2017, the hospice trained six of its staff as restorative facilitators. Those chosen were skilled up to an accredited level to help individuals or teams find their way out of conflict without following a formal grievance procedure.

What do our restorative facilitators do? Our restorative facilitators act as:

- an advisor - support individuals and help them to work out their options;

- a coach - help individuals practise how to frame their argument;

- a facilitator - help to facilitate a conversation where there is tension and conflict.

Outcomes The hospice's restorative facilitators work across the organisation, and this has led to a more positive, supportive culture. There have been no formal grievances undertaken since 2016 and feedback suggests that restorative practice has played a significant part in achieving this. Time has been saved and the hospice now has empowered managers and a happier more productive workforce whereby issues are discussed as and when they arise and staff are enabled to have direct, honest conversations with each other without fear of retribution.

Conclusions Restorative practice supports emotionally intelligent leadership, reduces formal grievances between staff and ultimately supports a positive organisational culture which has the potential to increase productivity and happiness at work.

\section{P-78 FEASIBILITY OF AN ONLINE TOOLKIT TO GUIDE IMPLEMENTATION OF THE CARER SUPPORT NEEDS ASSESSMENT TOOL}

${ }^{1}$ Janet Diffin, ${ }^{2}$ Gail Ewing, ${ }^{3}$ Christine Rowland, Gunn Grande ${ }^{3} .{ }^{1}$ Hospice UK, Belfast, UK; ${ }^{2}$ University of Cambridge, Cambridge, UK; ${ }^{3}$ University of Manchester, Manchester, UK

10.1136/bmispcare-2019-HUKNC.101

Background The Carer Support Needs Assessment Tool (CSNAT) intervention identifies and addresses the support needs of family carers. This carer-centred approach involves a change in practice from a practitioner to carer-led process of assessment and support. Training is needed to help practitioners transition to this new way of working, and implementation support is required at an organisational level (Austin, Ewing \& Grande, 2017; Diffin, Ewing, Harvey \& Grande, 2018; Diffin, Ewing, Harvey \& Grande, 2018). An online training and implementation toolkit was therefore developed to provide accessible and structured guidance on how to implement and embed the CSNAT intervention.

Aim To explore the feasibility and acceptability of delivering implementation training through an online format.

Methods The online toolkit has two learning components: (1) Individual: knowledge for practitioners to use the intervention, (2) Organisational: to assist a project team to plan, pilot and sustain implementation. Five UK palliative care services participated; 2-4 practitioners from each completed the toolkit. Online survey administered upon completion of each learning component for feedback on content, followed by telephone interview.

Results Fifteen practitioners completed 'Learning component 1' survey, 14 completed 'Learning component 2' survey, and 13 were interviewed. Feedback on content was positive. The most enjoyable aspects were the practical examples and the key steps to implementation being clearly detailed. The suitability of online learning was influenced by resource availability, the nature/size of the team, and individual learning styles. Variation in location of completion (work/home or both) was partly explained by availability of IT equipment, space, and time within the organisation. Whilst the majority of participants saw the value of online learning, some preferred faceto-face delivery, and so blended learning to include group discussions was deemed appropriate.

Conclusions Whilst online learning is welcomed by practitioners, organisations need to give it the same priority as attendance at face-to-face workshops and ensure sufficient resources are available to enable completion. Blended learning may help accommodate different learning preferences.

Funder NIHR CLARHC Greater Manchester.

\section{P-79 LESSONS FOR COMPREHENSIVE, PERSON-CENTRED CARER ASSESSMENT AND SUPPORT FROM THE CSNAT INTERVENTION}

${ }^{1}$ Gail Ewing, ${ }^{2}$ Gunn Grande, ${ }^{3}$ Janet Diffin, ${ }^{2}$ Lynn Austin. ${ }^{1}$ University of Cambridge, Cambridge, UK; ${ }^{2}$ University of Manchester, Manchester, UK; ${ }^{3}$ Project ECHOTM, Hospice UK, Belfast, UK

\subsection{6/bmispcare-2019-HUKNC.102}

Background The Carer Support Needs Assessment Tool (CSNAT) intervention comprises an evidence-based comprehensive tool incorporated into a person-centred process of assessment and support for carers of people with life-limiting conditions. It was developed from a programme of research, subsequently implemented across practice settings (community, hospital, hospice). The CSNAT's impact nationally and internationally is substantial: $87 \mathrm{UK}$ services are licensed to use the intervention, the tool has been translated into 13 languages, and is used in 27 countries.

Aim To draw on the CSNAT intervention programme of research to illustrate why carer assessment needs to be comprehensive and person-centred, how this represents a change in practice, what difference it makes, and how this change in practice can be implemented.

Methods/results We will draw on three aspects of the research programme:

1. An overview of development, validation and initial implementation of the CSNAT: a qualitative study with 75 bereaved carers (01/2008-12/2008) identified the 14 support need domains that constitute the CSNAT; a survey of 225 current carers (04/2009-06/2010) established CSNAT's content and criterion validity; implementation studies with 29 practitioners in two hospices (08/2010-12/2010; 01/2011-04/ 2012) identified use of the CSNAT as a significant change in practice and the importance of adopting a person-centred approach.

2. Two cluster randomised control trials of the CSNAT intervention (UK: $n=681$ carers; 05/2012-11/2014); (Australia: $\mathrm{n}=322$ carers; 03/2012-02/2014) showed a reduction in caregiver strain in current carers, and lower early grief and better physical/mental health in bereavement.

3. A national implementation study in 36 organisations (11/ 2013-09/2014) and a hospice case study (11/2015-12/2016) provided key insights into practitioners' training needs and vital organisational structures/processes needed to embed the intervention in practice, to underpin a CSNAT intervention Training and Implementation Toolkit. 